\title{
Activation of the Plasma Kallikrein-Kinin System in Respiratory Distress Syndrome
}

\author{
OLA D. SAUGSTAD, LAILA BU $\emptyset$, HARALD T. JOHANSEN, OLAV R $\emptyset$ ISE, AND \\ ANSGAR O. AASEN \\ Department of Pediatrics and Pediatric Research [O.D.S.J, Institute for Surgical Research, University of Oslo \\ [L.B., A.O.A.], Rikshospitalet, N-0027 Oslo 1, Department of Surgery [O.R.], Oslo City Hospital Ulleväl \\ University Hospital, N-0407 Oslo 4, Department of Pharmacology [H.T.J.], Institute of Pharmacy. \\ University of Oslo, $\mathrm{N}-0316$ Oslo 3, Norway
}

\begin{abstract}
Components of the plasma kallikrein-kinin and fibrinolytic systems together with antithrombin III were measured the first days postpartum in 13 premature babies with severe respiratory distress syndrome (RDS). Seven of the patients received a single dose of porcine surfactant (Curosurf) as rescue treatment. Nine premature babies without lung disease or any other complicating disease served as controls. There were no differences in prekallikrein values between surfactant treated and nontreated RDS babies during the first $4 \mathrm{~d}$ postpartum. The controls had, however, significantly higher prekallikrein values than the RDS babies already at the first day of age (mean \pm SD $32 \pm 8 \%$ in controls versus $22 \pm 6$ and 21.5 $\pm 5 \%$ in the treated and nontreated RDS groups, respectively). Plasma kallikrein activities did not differ between RDS and control patients. Plasma kallikrein inhibition values, which increased steadily in all groups, were lower in the RDS babies treated with surfactant than in controls at $\mathrm{d} 2$ and 4 . The degree of degradation of plasma high molecular weight kininogen was measured in RDS patients treated with surfactant and was significantly higher when compared with controls at $d \mathbf{1}$, demonstrating an increased proteolysis of kininogen to kinin early in RDS. There were no differences in plasminogen and plasmin values between RDS and control babies. This study shows that the plasma kallikrein-kinin system is activated in RDS. This system as well as the fibrinolytic system does not seem to be influenced by rescue instillation of a single dose of porcine surfactant into the lungs of premature babies with RDS. (Pediatr Res 32: 431-435, 1992)
\end{abstract}

\section{Abbreviations}

$\mathrm{FiO}_{2}$, fraction of oxygen in inspired air FXII, Hageman factor

HK, high molecular weight kininogen

RDS, respiratory distress syndrome

$\mathrm{PaO}_{2}$, oxygen tension in arterial blood

Activation of the proteolytic cascade systems of plasma is associated with trauma, septicemia, adult RDS, and neonatal RDS (1-6). The contact system of plasma comprises a set of

Received December 12, 1991; accepted May 11, 1992.

Correspondence: Prof. Ola D. Saugstad, M.D., Department of Pediatric Research, Rikshospitalet, N-0027 Oslo 1, Norway.

The surfactant (Curosurf) used in this study was developed by Tore Curstedt and Bengt Robertson, Karolinska Institutet, Stockholm, with financial support from The Swedish Medical Research Council (project No. 3351) and Oscar Il:s Jubileumsfond. proteins that interact in a complicated way. When activated, the contact factors plasma prekallikrein, FXII, and factor XI are converted to serine proteases that are capable of activating the complement, fibrinolytic, coagulation, and kallikrein-kinin systems (7-9). Inhibitors regulate and control the activation of the cascades. C1-inhibitor is the most important inhibitor of the contact system (10). It exerts its regulatory role by inhibiting activated FXII, FXII fragment, and plasma kallikrein (10). In addition, $\alpha_{2}$-macroglobulin and $\alpha_{1}$-protease inhibitor inhibit plasma kallikrein (11) and factor XIa (12), respectively. Contact activation initiates other proteolytic plasma cascades. Plasma prekallikrein, activated FXII, and factor XIa may convert the proenzyme plasminogen to plasmin $(13,14)$.

When the plasma kallikrein-kinin system is activated, $\mathrm{HK}$ is cleaved proteolytically and three characteristic bands are identified by electrophoresis followed by immunoblotting. The relative amount of each of these bands gives an indication of the degree of plasma kallikrein-kinin activation. Band I with molecular mass of $149000 \mathrm{D}$ represents the native form of $\mathrm{HK}$; band II (molecular mass 104000 D) and band III (molecular mass 99000 D) are considered to represent degradation products of kininogen. The relative amounts of these two compounds are therefore of special interest when considering the rate of proteolytic cleavage of kininogen (15).

The plasma kallikrein-kinin system may play a fundamental role in the development of shock-like conditions because its activation results in the formation of the very potent vasodilatator bradykinin, which increases capillary permeability as well $(7,9,16,17)$. We have previously shown that preterm babies with RDS have low plasma prekallikrein and kallikrein inhibition levels, indicating that the plasma kallikrein-kinin system might be activated in this condition (6).

The purpose of the present study was to investigate more specifically whether or not the plasma kallikrein-kinin system is activated in RDS. In addition to components of the plasma kallikrein-kinin system including HK and its degradation products, we have also determined components of the fibrinolytic system and antithrombin III in preterm babies with and without RDS. Additionally, we have studied whether rescue treatment with a single dose of porcine surfactant influences the activation of these systems.

\section{MATERIALS AND METHODS}

Patients. The study population comprised 22 preterm babies divided into three groups. Gestational age ranged between 25 and $33 \mathrm{wk}$ and birth weight between 795 and $1785 \mathrm{~g}$. Group 1 $(n=7)$ and group $2(n=6)$ babies had RDS diagnosed according to well-established clinical and radiographic criteria (18). Only babies with severe RDS defined as oxygen requirement $\geq 0.60$ and dependence on artificial ventilation were included. Group 1 
babies received one dose of natural surfactant as rescue treatment. Mean gestational age in this group of patients was 28.5 (SD 0.8) wk and birth weight was 1241 (SD 134) g. Group 2 babies were not treated with surfactant. They had a mean gestational age of 28.8 (SD 3.2) wk and birth weight of 1197 (SD 349) $\mathrm{g}$.

The babies with RDS fulfilled the criteria for entering the first Collaborative European Multicenter Trial of replacement therapy with porcine surfactant (Curosurf) (19). The protocol and results from this study have previously been published in detail (19). In that study, RDS babies were randomized either to receive or not to receive a single dose of surfactant.

Group $3(n=9)$ consisted of controls without RDS or any other significant respiratory problems. To be enrolled as controls, the babies did not have an oxygen requirement $\geq 0.30$ for more than $1 \mathrm{~h}$ after birth and required artificial ventilation only for stabilization during the first $2 \mathrm{~d}$ after birth. They had a mean gestational age of 28.9 (SD 1.8) wk and birth weight of 1138 (SD 207) $\mathrm{g}$. The mothers of three of them received dexamethasone between 1 and $7 \mathrm{~d}$ before giving birth. Eight of nine control babies were delivered by cesarean section. Their mean 1-min Apgar score was 6.7 (range 2-9), and the 5-min score was 8.4 (range 6-10). Four of these babies required intermittent positive ventilation of duration ranging between 1 and $36 \mathrm{~h}$ postpartum.

There were no significant differences between the three groups with regard to birth weight and gestational age. All babies were larger than the 2.5 percentile for weight.

Procedure. An arterial or venous umbilical catheter was inserted for clinical monitoring; the routine in our unit is to remove such indwelling catheters within $96 \mathrm{~h}$ if the babies do not require artificial ventilation.

All the RDS babies had indwelling arterial umbilical catheters, which were removed at latest approximately $24 \mathrm{~h}$ after extubation. Blood for analysis was withdrawn as soon as possible after stabilization after birth. The first blood sample (d 1) was withdrawn before the age of $12 \mathrm{~h}$ at a mean age of 7.5 (SD 3.0) $\mathrm{h}$ and then at 24-h intervals as long as an umbilical catheter remained in position. For babies receiving surfactant, the first blood sample was withdrawn $5 \mathrm{~min}$ before surfactant was given. All three groups were treated identically with regard to timing of blood sampling.

Catheters in control babies were removed between 72 and 96 $\mathrm{h}$ of age and data are therefore given only until $\mathrm{d} 4 \mathrm{after}$ birth.

From the catheter, $0.9 \mathrm{~mL}$ of blood was withdrawn, avoiding contact with any heparin, and put into soft plastic tubes with 0.1 $\mathrm{mL} 3.2 \%$ citrate. After centrifugation, plasma was sucked off and frozen at $-20^{\circ} \mathrm{C}$ until assayed.

Measurements. Plasma kallikrein activity, prekallikrein, and functional kallikrein inhibition values were determined with the chromogenic peptide substrate S-2302 (Kabi, Stockholm, Sweden) using an automated enzyme analyzer (Cobas Bio, Hoffmann La Roche, Basel, Switzerland) as previously described (20). The chromogenic substrate S-2251 (Kabi) was used to determine plasminogen, plasmin and antiplasmin values. The values of prekallikrein, kallikrein inhibition, plasminogen, and antiplasmin are given as percentages of standard plasma pool values. Antithrombin III values were assayed with the chromogenic substrate S-2238 (Kabi) as previously described (20). Plasma total protein was measured with a routine technique (21).

HK and its degradation products were determined by SDS PAGE followed by immunoblotting according to standard procedures $(22,23)$. The blot was washed, quenched, and incubated with rabbit antiserum against human HK. Bound antibodies were finally detected with alkaline phosphatase-conjugated goat antibodies against rabbit $\mathrm{IgG}$. The developed immunoblots were scanned and the area under the curve for each band was used as a measure of the relative amount of native kininogen or degradation product (24).

The project was approved by the ethical committee of the hospital, and informed consent was obtained from the parents of the babies. Statistical differences between the groups were tested by a two-tailed $t$ test. A $p$ value $<0.05$ was considered statistically significant.

\section{RESULTS}

Oxygenation requirements and $\mathrm{PaO}_{2}$. In Figure $1, \mathrm{FiO}_{2}$ in the three patient groups is given until $\mathrm{d} 4$ after birth. The mean initial $\mathrm{FiO}_{2}$ was 0.91 (SD 0.09) in group 1 (RDS surfactant) and 0.94 (SD 0.09) in group 2 (RDS patients without surfactant) (NS). On d 2 ( $24 \mathrm{~h}$ after surfactant instillation in group 1 babies), the $\mathrm{FiO}_{2}$ was, however, significantly lower in surfactant-treated RDS patients (mean 0.38, SD 0.08) compared with nontreated RDS patients (mean 0.82, SD 0.08) $(p<0.04)$. Mean $\mathrm{FiO}_{2}$ remained lower in the surfactant group also at $\mathrm{d} 3$ (mean 0.43 , SD 0.22) compared with nontreated RDS babies (mean 0.80, SD $0.13)(p<0.05)$. On d 4, mean $\mathrm{FiO}_{2}$ in surfactant-treated babies was 0.42 (SD 0.07) compared with 0.63 (SD 0.18) in nontreated RDS babies (NS).

The mean $\mathrm{FiO}_{2}$ in the controls was initially 0.26 (SD 0.04), ranging between 0.21 and 0.30 , significantly lower than in both of the RDS groups. After $48 \mathrm{~h}$, eight of the nine controls breathed room air.

$\mathrm{PaO}_{2}$ was initially 7.4 (SD 2.0) $\mathrm{kPa}$ in the surfactant-treated group (group 1) versus 10.1 (SD 4.1) $\mathrm{kPa}$ in group 2 (NS). During the study, mean $\mathrm{PaO}_{2}$ in the RDS patients with and without surfactant treatment were not statistically different and varied between 6.5 and $10.1 \mathrm{kPa}$.

Plasma prekallikrein, kallikrein, and kallikrein inhibition. Plasma prekallikrein values are given in Figure 2. There were no differences in prekallikrein values between the surfactant-treated (group 1) and nontreated RDS (group 2) babies during the study. In the first plasma sample (d 1), the mean prekallikrein values were 22 (SD 6)\% in group 1 ( $p<0.005$ versus controls), 21.5 (SD 5.0)\% in group 2 ( $p<0.0015$ versus controls), and 32 (SD $8) \%$ in the control group (group 3). Twenty-four h later (d 2), there was still no difference between the two RDS groups, but the differences between these two groups and the controls were even larger, with a mean of 20 (SD 5)\% in group $1(p<0.0002)$, 21 (SD 6)\% in group $2(p<0.0005)$, and 37 (SD 7)\% in controls. The next $2 \mathrm{~d}$ prekallikrein values increased steadily and parallelly in all groups, reaching at d 4 a mean of 31 (SD 6)\% in group 1 ( $p<0.05$ versus controls), 27 (SD 4)\% in group $2(p<0.02$ versus controls), and 47 (SD 15$) \%$ in controls.

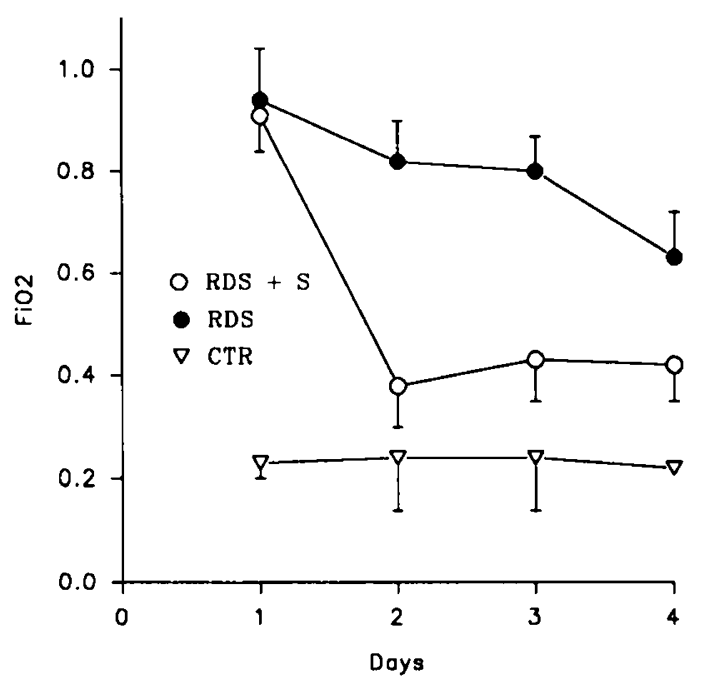

Fig. 1. $\mathrm{FiO}_{2}$ in three groups of patients the first $4 \mathrm{~d}$ after birth. $R D S$ $+S$, RDS patients treated with a single dose of natural surfactant 5 min after the reading at d 1.RDS, RDS patients not treated with surfactant. $C T R$, premature babies without RDS who served as controls. Mean and $\mathrm{SD}$ are given as range. 


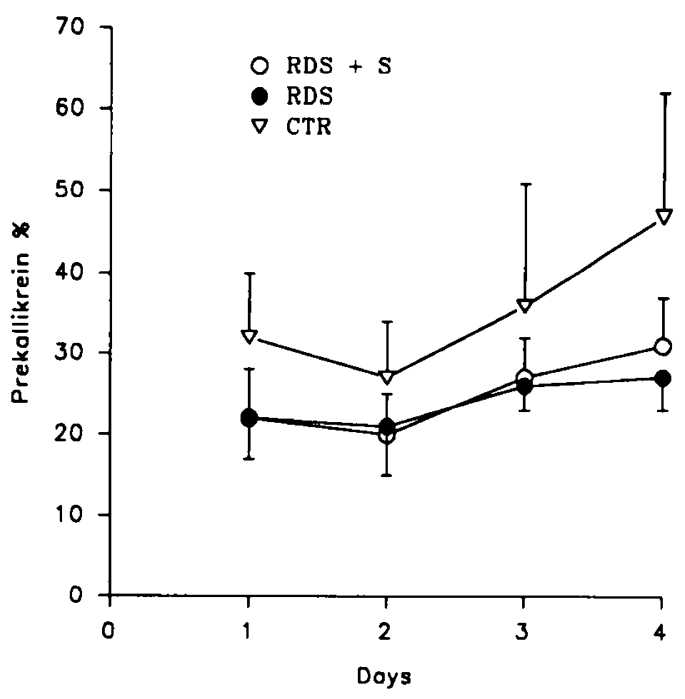

Fig. 2. Plasma prekallikrein values as the percentage of standard plasma pool values the first $4 \mathrm{~d}$ after birth. $R D S+S$, RDS patients treated with a single dose of natural surfactant 5 min after the reading at d 1. $R D S$, RDS patients not treated with surfactant. $C T R$, premature babies without RDS who served as controls. Mean and SD are given as range.

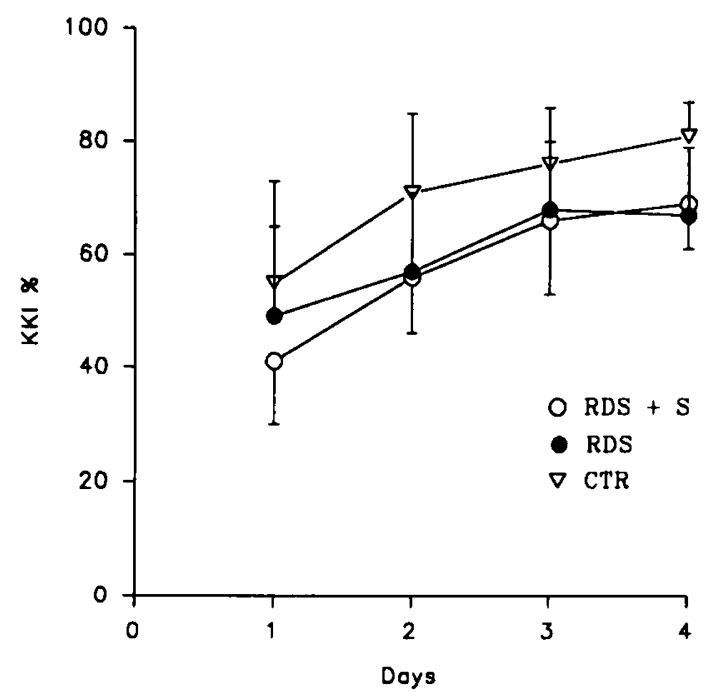

Fig. 3. Plasma kallikrein inhibition $(K K I)$ values as the percentage of standard plasma pool values the first $4 \mathrm{~d}$ after birth. $R D S+S$, RDS patients treated with a single dose of natural surfactant 5 min after the reading at d 1. RDS, RDS patients not treated with surfactant. CTR, premature babies without RDS who served as controls. Mean and SD are given as range.

The plasma kallikrein activity was low but stable, varying between 8 and $10 \mathrm{U} / \mathrm{L}$ in the two groups of RDS babies and between 7 and $12 \mathrm{U} / \mathrm{L}$ in the controls during the first $4 \mathrm{~d}$ after birth. No significant differences between the three groups were found.

Plasma kallikrein inhibition values increased in all the three groups during the study period (Fig. 3). There were no differences between groups 1 and 2 throughout the observation period. Group 1 babies had, however, signficantly lower values than controls at $d 2(p<0.02)$ and $4(p<0.05)$, whereas group 2 patients had values not significantly different from controls.

Proteolysis of $H K$. To study proteolytic activity in more detail, HK (band I) and its degradation products (bands II and III) were measured in RDS patients receiving surfactant (group 1) and compared with the controls at $\mathrm{d} 1$ and 2 . The mean total amount of kininogen (sum of bands I, II, and III) measured as area under the curve was 5081 (SD 2327 ) in group 1 and 8222 (SD 3612 ) in controls at $\mathrm{d} 1$ (NS, $p<0.09$ ). At $\mathrm{d} 2$, the values were 4250 (SD 3 993) versus 13279 (SD 19000) (NS), respectively.

Band I values were significantly lower in group 1 patients at $d$ 1 (mean 2022 , SD 1365 ) than in controls (mean 4370 , SD 2 203) $(p<0.04)$. Band III (Fig. 4) values were equal in groups 1 and 3 at $d 1$. In group 1 babies, there was a dramatic decrease from a mean value of 1163 (SD 530) to 150 (SD 34) $(p<$ 0.0025 ) from $d 1$ to $d 2$. In controls, the amount of band III remained stable.

Figure 5 shows a SDS-PAGE immunoblot of HK and its degradation products in one RDS patient treated with surfactant the first week after birth. Band III, which is the most important indicator of $\mathrm{HK}$ proteolysis, was clearly present at d 1 . After $\mathrm{d} 5$ it disappeared completely.

Figure 6 demonstrates scanning of HK and its degradation products in the same patient at $\mathrm{d} 1$ (left) and d 2 (right). It can be seen how band III is clearly present the first day and that the amount decreases until the next day. This indicates a reduced proteolysis of HK from $\mathrm{d} 1$ to $\mathrm{d} 2$.

These data strongly indicate that the rate of proteolysis of HK was slowed down from $\mathrm{d} 1$ to $\mathrm{d} 2$ in RDS patients.

The fibrinolytic system and antithrombin III. The results of the measurements of plasminogen, plasmin, and antiplasmin at d 1-4 in the three groups of patients are given in Table 1 . There were no differences between the three groups except with regard

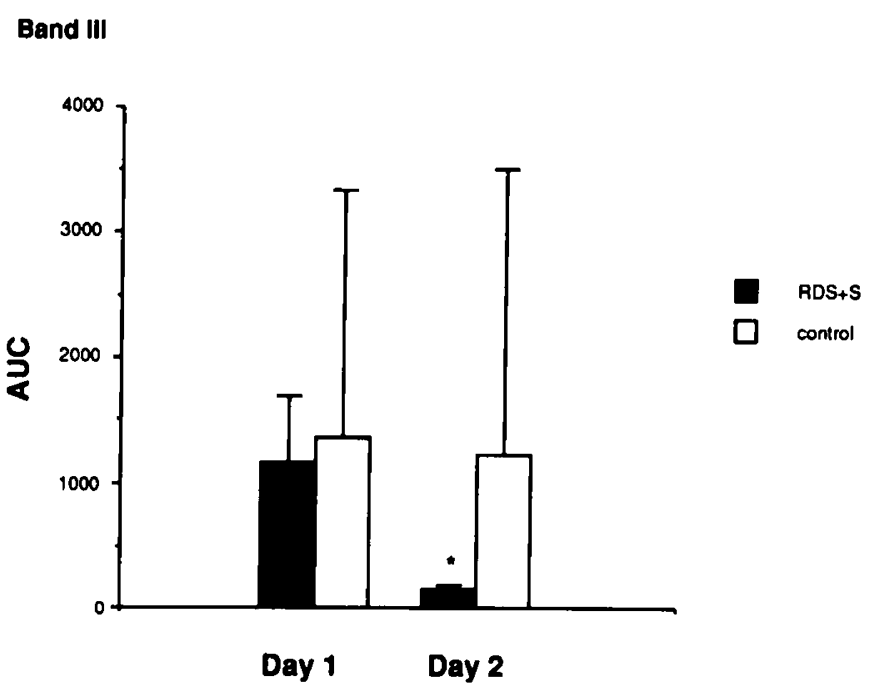

Fig. 4. The change in proteolysis of HK from $\mathrm{d} 1$ to $\mathrm{d} 2$ in babies with RDS treated with a single dose of surfactant $(R D S+S)$ compared with premature babies without RDS serving as controls. Rate of proteolysis is illustrated by the relative amount of degradation product detected as band III. $A U C$, area under the curve. $\mathrm{SD}$ is given as range. ${ }^{*}, p<$ $0.0025 \mathrm{RDS}+\mathrm{S}$ at $\mathrm{d} 1$ vs $\mathrm{d} 2$.

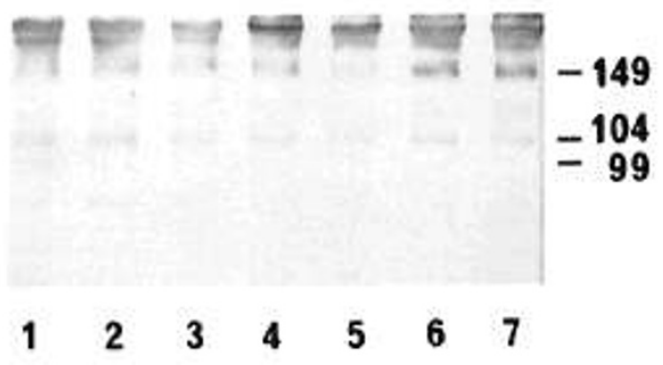

Fig. 5. Immunoblot of HK at $d$ to 7 after birth in one patient with RDS treated with a single dose of surfactant. Band I (molecular mass $149000 \mathrm{D}$ ) represents HK, band II (molecular mass $104000 \mathrm{D}$ ) and band III (molecular mass $99000 \mathrm{D}$ ) represent proteolytic breakdown products of HK. Band III is clearly present at d 1. At d 6 and 7, it has completely disappeared. 


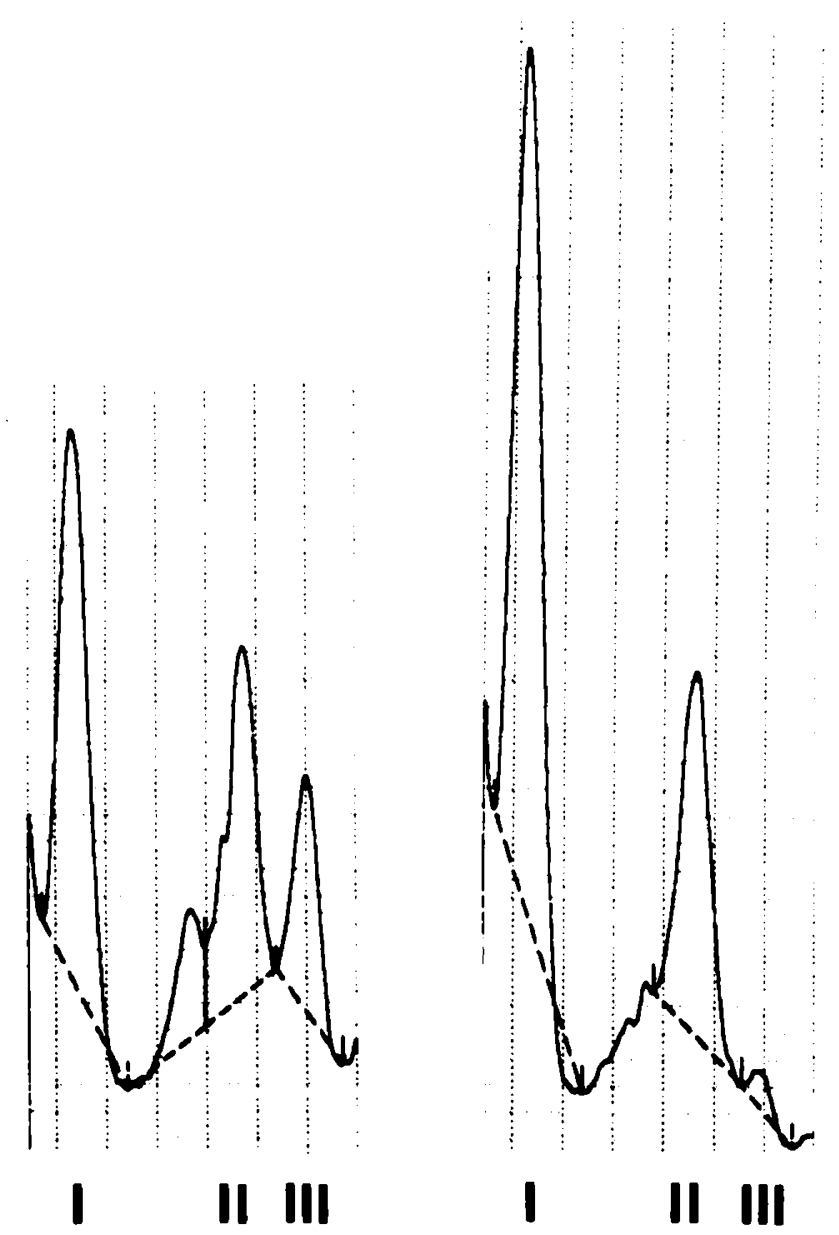

Fig. 6. Scanning of the three bands derived from HK at $d 1$ and 2 in the same RDS patient as in Figure 4. Band III clearly is diminished from d 1 to $\mathrm{d} 2$, demonstrating reduced proteolytic conversion of HK.

Table 1. Data from fibrinolytic system for first $4 d$ after birth*

\begin{tabular}{cllll} 
& \multicolumn{4}{c}{ Day } \\
\cline { 2 - 5 } & \multicolumn{1}{c}{1} & \multicolumn{1}{c}{2} & \multicolumn{1}{c}{3} & \multicolumn{1}{c}{4} \\
\hline Plasminogen (\%) & & & & \\
$\quad$ Group 1 & $31(10)$ & $31(6)$ & $36(10)$ & $38(9)$ \\
$\quad$ Group 2 & $31(10)$ & $31(9)$ & $34(9)$ & $34(11)$ \\
$\quad$ Group 3 & $32(15)$ & $37(11)$ & $39(14)$ & $50(20)$ \\
Plasmin (U/L) & & & & \\
Group 1 & $10(7)$ & $15(9)$ & $22(17)$ & $10(7)$ \\
$\quad$ Group 2 & $15(9)$ & $21(16)$ & $27(18)$ & $29(23)$ \\
Group 3 & $15(12)$ & $25(15)$ & $18(9)$ & $12(8)$ \\
Antiplasmin (\%) & & & & \\
$\quad$ Group 1 & $58(13)$ & $67(10)$ & $72(9)$ & $75(9)$ \\
$\quad$ Group 2 & $40(27)$ & $43(26) \dagger$ & $48(29)$ & $42(26)$ \\
Group 3 & $62(23)$ & $75(13)$ & $82(19)$ & $90(22)$ \\
\hline
\end{tabular}

* Group 1, RDS patients treated with porcine surfactant; Group 2, RDS patients not treated with surfactant; Group 3, premature control babies without RDS. Plasminogen and antiplasmin are given as the percentage of standard pooled plasma values. Mean values and SD (numbers in parentheses) are given.

$\dagger p<0.03$, RDS $v s$ controls.

to plasma antiplasmin values, which at $\mathrm{d} 2$ were significantly lower in the group 2 RDS babies than in controls.

Antithrombin III values were low compared to pool plasma values from normal adults, but there was neither any difference between the groups nor any significant change during the study (data not shown).

Plasma total protein. In control babies, plasma total protein at $\mathrm{d} 1$ and 2 were 39.6 (SD 1.8) $\mathrm{g} / \mathrm{L}$ and 41.5 (SD 3.1) g/L, respectively. Plasma total protein was not determined in these babies later on. Plasma total protein did not differ between the the two RDS groups, and the mean value was 35.3 (SD 3.4) $\mathrm{g} / \mathrm{L}$ at $\mathrm{d} \mathrm{I}$, which is significantly lower than values found for control babies $(p<0.04)$. The next $3 \mathrm{~d}$, plasma total protein concentrations in RDS patients were stable, with mean values ranging between 37 and $38 \mathrm{~g} / \mathrm{L}$.

\section{DISCUSSION}

In the present study, we have demonstrated that plasma prekallikrein values were significantly lower in babies with severe RDS compared with control babies without RDS the first days after birth. These data are basically in accordance with our previous findings (6). During the first day of life, mean plasma prekallikrein in both groups of RDS patients was $63 \%$ of the values found in premature control patients without RDS. Although the plasma total protein was decreased in RDS babies as well, this reduction was, in mean, only $11 \%$ compared with control values. The differences in prekallikrein values found between the groups in this study is therefore probably not due to a general decreased protein synthesis in the liver in RDS babies. On the contrary, the data indicate that the plasma kallikreinkinin system is activated in RDS.

The low HK levels found at $\mathrm{d} 1$ in RDS patients, together with the rapid decrease in the amount of its degradation products (band III) from $\mathrm{d} 1$ to $\mathrm{d} 2$, as found in this study, represent the first direct proof that the plasma kallikrein-kinin system is activated in RDS. Rescue treatment with porcine surfactant does not seem to enforce or prevent this activation or influence the fibrinolytic system either. Whether or not prophylactic surfactant treatment inhibits activation of the plasma kallikrein-kinin system is not known and should be worthwhile to investigate.

Judging by plasminogen values and plasmin activity, the fibrinolytic system is not activated in RDS, in contrast to findings by Suzuki (25).

It has been previously demonstrated that plasma prekallikrein is transformed to kallikrein in a series of other pathophysiologic conditions such as septicemia, multitraumatized patients with multiple organ failure, and the adult RDS $(1-5,17)$. On the basis of the present data it may therefore be tempting to speculate that RDS has some features in common with "posttraumatic" diseases such as those listed above (1-5). It is known that RDS may be aggravated and precipitated by shock, hypothermia, and acidosis $(26,27)$. To what extent activation of the plasma kallikrein-kinin system influences the clinical development in patients with RDS is, however, uncertain.

In conclusion, we have found lowered plasma prekallikrein and kallikrein inhibition values in babies with RDS. The first day after birth, the total amount of HK is reduced in these patients compared with premature babies not having RDS. Furthermore, the rate of proteolytic degradation of HK is reduced in RDS babies from the first to the second day of life. We have therefore documented that the kallikrein-kinin system is activated in RDS. Rescue treatment with one dose of natural porcine surfactant does not seem to influence such an activation.

Acknowledgments. The authors thank Tore Curstedt and Bengt Robertson for contributing valuable ideas and support to the present study.

\section{REFERENCES}

I. Risberg B, Medegä A, Heideman M, Gyzander E, Bundsen P, Oden M, TegerNilsson A-C 1986 Early activation of humoral proteolytic systems in patients with multiple trauma. Crit Care Med 14:917-925

2. Aasen AO, Smith-Erichsen N. Amundsen E 1986 Uncontrolled plasma proteolysis: a major threat to the septicemic patient. Resuscitation 14:33-42

3. Aasen AO, Rishovd A-L, Stadaas JO 1989 Role of endotoxin and proteolysis in multiple organ failure (MOF). Prog Clin Biol Res 308:315-322

4. Lammle B, Tran TH, Ritz R, Ducker F 1984 Plasma prekallikrein, factor XII, 
antithrombin III, $\mathrm{Cl}$-inhibitor and $\alpha_{2}$-macroglobulin in critically ill patients with suspected disseminated intravascular coagulation (DIC). Am J Clin Pathol 82:396-404

5. Carvhalho AC, Demarinis S, Scott CF, Silver LD, Schmaier AH, Colman RW 1988 Activation of the contact system of plasma proteolysis in the adult respiratory distress syndrome. J Lab Clin Med 112:270-277

6. Saugstad OD, Harvie A, Langslet A 1982 Activation of the kallikrein-kinin system in premature infants with respiratory distress syndrome (RDS). Acta Paediatr Scand 71:965-968

7. Cochrane CG, Griffin JH 1979 Molecular assembly in the contact phase of the Hageman factor system. Am J Med 67:657-664

8. Cochrane CG, Griffin JH 1985 The biochemistry and pathophysiology of the contact system of plasma. In: Dixon FJ, Kunkel HG (eds) Advances in Immunology. Academic Press, New York, pp 241-306

9. Colman RW, Schmaier AH 1986 The contact activation system: biochemistry and interactions of these surface-mediated defense reactions. CRC Crit Rev Oncol Hematol 5:57-85

10. van der Graaf F, Koedam JA, Bouma BN 1983 Inactivation of kallikrein in human plasma. J Clin Invest 71:149-158

11. van der Graaf F, Rietvald A, Keus FJA, Bouma BN 1984 Interaction of human plasma kallikrein and its light chain with $\alpha_{2}$-macroglobulin. Biochem 23:1760-1766

12. Scott CF, Schapira M, James HL, Cohen AB, Colman RW 1982 Inactivation of factor Xla by plasma protease inhibitors. Predominant role of $\alpha_{1}$-protease inhibitor and protective effect of high molecular weight kininogen. J Clin Invest 69:844 852

13. Mandle R, Kaplan AP 1977 Hageman factor substrates. Human plasma prekallikrein: mechanism of activation by Hageman factor and participation in Hageman factor-dependent fibrinolysis. J Biol Chem 252:6097-6104

14. Goldsmith GH, Saito H, Ratnoff OD 1978 The activation of plasminogen by Hageman factor (factor XII) and Hageman factor fragments. J Clin Invest $62: 54-60$
15. Schmaier AH, Farber A, Schein R, Sprung C 1988 Structural changes of plasma high molecular weight kininogen after in vitro activation and in sepsis. J Lab Clin Med 112:182-192

16. Erdoes EG 1976 The kinins. A status report. Biochem Pharmacol 25:15631569

17. Aasen AO, Frolich W, Saugstad OD, Amundsen E 1978 Plasma kallikrein activity and prekallikrein levels during endotoxin shock in dogs. Eur Surg Res 10:50-62

18. Hjalmarson O 1981 Epidemiology and classification of acute, neonatal respiratory disorders: a prospective study. Acta Paediatr Scand 70:773-783

19. Collaborative European Multicenter Study Group 1988 Surfactant replacement therapy for severe neonatal respiratory distress syndrome: an international randomized clinical trial. Pediatrics 82:683-691

20. Aasen AO, Kierulf P, Stroeme JH 1982 Methodological considerations on chromogenic peptide substrate assays and applications on automated analysis. Acta Chir Scand Suppl 509:17-22

21. Kingsley GR 1939 The determination of serum total protein, albumin, and globulin by the biuret reaction. J Biol Chem 131:197-200

22. Laemmli UK 1970 Cleavage of structural proteins during the assembly of the head of bacteriophage T4. Nature 227:680-685

23. Towbin H, Staehelin T, Gordon J 1979 Electrophoretic transfer of proteins from polyacrylamide gels to nitrocellulose sheets: procedure and some applications. Proc Natl Acad Sci 76:4350-4354

24. Karlsrud, TS, Buф L, Aasen AO, Johansen HT 1991 Characterization of kininogens in human malignant ascites. Thrombosis Res 63:641-650

25. Suzuki S 1978 Blood coagulation and fibrinolysis of the newborn viewed as perinatal factors. J Perinat Med 6:45-58

26. Adams HF, Nozaki M, Chida N, Salaway AE, Norma A 1967 Effects of hypoxemia, hypercarbia, acidosis and reduced pulmonary flow on surfactant of fetal lamb lungs. J Pediatr 71:396-403

27. Merritt TA, Farell PM 1976 Diminished pulmonary lecithin synthesis in acidosis. Experimental findings as related to the respiratory distress syndrome. Pediatrics 40:32-40 